\title{
Recent results from the cosmic ray program of the NA61/SHINE experiment
}

\author{
Raul R. Prado ${ }^{1,2,3, *}$ for the NA61/SHINE Collaboration** \\ ${ }^{1}$ Deutsches Elektronen-Synchrotron (DESY), Platanenallee 6, D-15738 Zeuthen, Germany \\ ${ }^{2}$ IKP, Karlsruhe Institute of Technology (KIT), Postfach 3640, D-76021 Karlsruhe, Germany \\ ${ }^{3}$ Instituto de Física de São Carlos (IFSC/USP), Av. Trabalhador São-carlense 400, 13566-590, São Carlos, Brazil
}

\begin{abstract}
NA61/SHINE is a fixed target experiment designed to study hadron-proton, hadron-nucleus and nucleus-nucleus interactions at the CERN Super-Proton-Synchrotron. In this paper we summarize the results from pion-carbon collisions recorded at beam momenta of 158 and $350 \mathrm{GeV} / c$. Hadron production measurements in these types of interactions is of fundamental importance for the understanding of the muon production in extensive air showers. In particular, production of (anti)baryons and $\rho^{0}$ are mechanisms responsible for increasing the number of muons which reach the ground. The underestimation of the (anti)baryons or $\rho^{0}$ production rates in current hadronic interaction models could be one of the sources of the excess of muons observed by cosmic ray experiments. The results on the production spectra of $\pi^{ \pm}, \mathrm{K}^{ \pm}, \mathrm{p}, \overline{\mathrm{p}}, \Lambda, \bar{\Lambda}, \mathrm{K}_{\mathrm{S}}^{0}, \rho^{0}, \omega$ and $\mathrm{K}^{* 0}$ are presented, as well as their comparison to predictions of hadronic interaction models currently used in air shower simulations.
\end{abstract}

\section{Introduction}

Measurements of ultrahigh energy cosmic rays are only possible through the detection of secondary particles produced in extensive air showers (EAS). The inference of some of the properties of the primary cosmic ray particles like their nuclear mass, relies on the comparison of measured EAS observables to predictions from simulations [1]. These simulations are performed by Monte Carlo codes that make use of hadronic interaction models to describe the nucleus-air and hadron-air collisions along the shower development [2]. Although simulations using recent hadronic models can provide a good overall description of EAS, it has been observed by cosmic ray experiments that the hadronic models fail on describing the muon production in EAS. Measurements by HiResMIA [3], Pierre Auger Observatory [4-7], Telescope Array [8], KASCADE-Grande [9], IceTop/IceCube [10] and Sugar [11] show that there is an inconsistency between data and simulations for observables related to the muonic component of air showers. In particular, the number of muons $\left(N_{\mu}\right)$ obtained from simulations is observed to be significantly smaller than the measured ones, which is known as the "muon deficit problem".

The majority of muons in EAS are produced by the decay of charged mesons, which in turn, are produced in meson-air and nucleon-air interactions. Depending on the primary energy and detection distance, the relevant mesonair and nucleon-air interaction energies are between 10 and $1000 \mathrm{GeV}[12,13]$. Therefore, measurements of particle production in this energy range are of great value for understanding muon production in EAS and consequently for improving its modeling. Of particular interest are the pro-

\footnotetext{
*e-mail: raul.prado@desy.de

${ }^{* *}$ Full author list: http://shine.web.cern.ch/content/author-list
}

duction spectra of (anti-)baryons and $\rho^{0}$ in meson-air and nucleon-air. It is well known $[14,15]$ that the production of (anti-)baryons and $\rho^{0}$ mesons in hadronic interactions is important to predict the muon content of air showers. Therefore the production cross sections of these particles needs to be known accurately for a precise modeling of air showers

NA61/SHINE experiment [16] (see Sec. 2) has provided a number of particle production and cross section measurements which are relevant for the modeling of hadronic interaction in EAS (e.g. Refs [17, 18]). In this paper, however, the focus will be on the results of the 2009 run with a negatively charged pion beam colliding against a thin carbon target $\left(\pi^{-}+\mathrm{C}\right.$ data) at 158 and $350 \mathrm{GeV} / c$. Since $\pi$-air is the most abundant hadronic interaction occurring in an EAS, our $\pi^{-}+\mathrm{C}$ data is of high relevance for the tuning of hadronic interaction models dedicated to EAS simulations. The results are presented in three parts: the spectra of charged hadrons $\left(\pi^{ \pm}, K^{ \pm}, p\right.$ and $\left.\bar{p}\right)$ are presented in Sec. 3, the spectra of $V^{0}$ mesons $\left(\Lambda, \bar{\Lambda}\right.$ and $\left.\mathrm{K}_{\mathrm{S}}^{0}\right)$ in Sec. 4 and the spectra of resonance mesons $\left(\rho^{0}, \mathrm{~K}^{* 0}\right.$ and $\left.\omega\right)$ in Sec. 5 .

\section{The NA61/SHINE experiment}

NA61/SHINE (SHINE = SPS Heavy Ion and Neutrino Experiment) is a fixed target experiment at the CERN SPS designed to study hadron production in nucleus-nucleus and hadron-nucleus collisions. Its physics goals comprise a) the strong interaction program, which investigates the properties of the onset of deconfinement and search for the critical point of strongly interacting matter, b) the neutrino program, to precisely measure the hadron production important to calculate the neutrino and antineutrino fluxes in the T2K neutrino experiment [19], and c) the cosmic ray program, focused on the measurements of the hadron and 


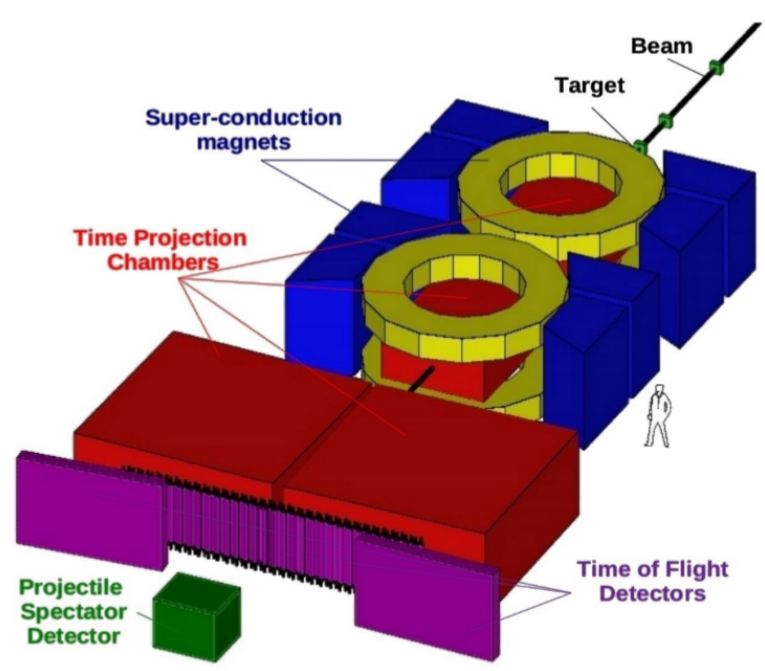

Figure 1: Schematic layout of the NA61/SHINE experiment [16].

meson production which are most relevant for the modeling of extensive air showers. The full description of the NA61/SHINE experiment and its science program can be found in Ref. [16]

The NA61/SHINE detector measures charged particles produced by the collision of the beam particles with the target through a set of five Time Projection Chambers (TPC). Since two of the TPCs are placed in the magnetic field produced by superconducting dipole magnets, the charge and the momenta of the particles can be measured and the achieved resolution on $p$ is of the order of $\sigma(p) / p^{2}=10^{-4}$ $(\mathrm{GeV} / c)^{-1}$. Additionally, the energy loss per unit of length $(\mathrm{d} E / \mathrm{d} x)$ in the TPCs is used in this work for particle identification. The experimental layout of the NA61/SHINE detector is shown in Fig. 1.

A beam detector system composed of scintillation and Cherenkov counters is placed upstream of the detector to identify and measure the beam particles. The position of the beam is measured by a set of three beam position detectors, which are also placed upstream of the target.

\section{Production of $\pi^{ \pm}, K^{ \pm}, p$ and $\bar{p}$}

Charged particles are identified in NA61/SHINE by the track-by-track measurement of the deposited energy, $\mathrm{d} E / \mathrm{d} x$, performed by the TPCs. After splitting the data into bins of total and transverse momentum ( $p$ and $p_{\mathrm{T}}$ ), a $\mathrm{d} E / \mathrm{d} x$ model is fitted to the measured $\mathrm{d} E / \mathrm{d} x$ distributions by accounting for contributions of 5 particle types $(e, \pi$, $K, p$ and deuterons). From the results of the fit, the particle yields of $\pi^{ \pm}, \mathrm{K}^{ \pm}$and $\mathrm{p}(\overline{\mathrm{p}})$ are determined. Examples of measured $\mathrm{d} E / \mathrm{d} x$ distributions and of the results of the $\mathrm{d} E / \mathrm{d} x$ fit are shown in Fig. 2. After performing the particle identification through the $\mathrm{d} E / \mathrm{d} x$ fit, the detector effects (e.g. acceptance, efficiency) are corrected by using a set of Monte Carlo simulations and the spectra are derived. A more detailed description of the analysis procedure can be found in Ref. [20].

The single-differential spectra as a function of $p$ (integrated over $\left.p_{\mathrm{T}}\right)$ for $\pi^{ \pm}, \mathrm{K}^{ \pm}$and $\mathrm{p}(\overline{\mathrm{p}})$ are shown in Figs. 5

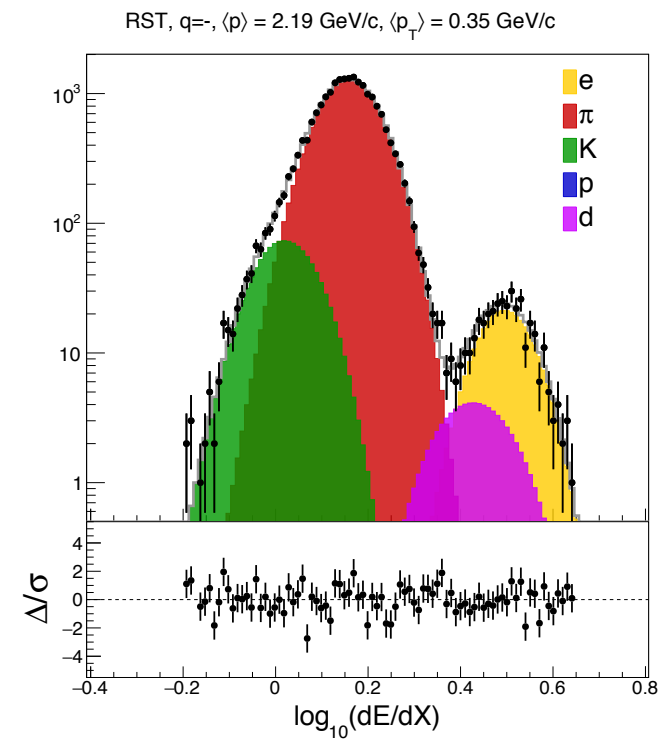

RST, $q=+,\langle p\rangle=2.19 \mathrm{GeV} / \mathrm{c},\left\langle\mathrm{p}_{\mathrm{T}}\right\rangle=0.35 \mathrm{GeV} / \mathrm{c}$

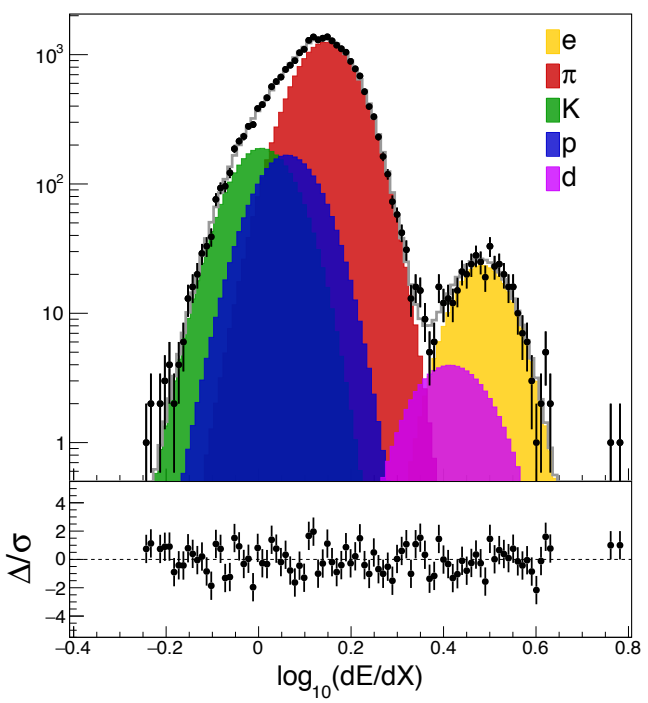

Figure 2: Example of the $\mathrm{d} E / \mathrm{d} x$ distributions for one phase space bin $\left(\langle p\rangle=2.19 \mathrm{GeV} / c\right.$ and $\left.\left\langle p_{\mathrm{T}}\right\rangle=0.35 \mathrm{GeV} / c\right)$ of the $158 \mathrm{GeV} / c$ data set. The black markers show the measured distributions and the colored distributions show the result of the $\mathrm{d} E / \mathrm{d} x$ fit. Negatively charged particles are shown on the top and positively ones on the bottom.

and 6, where the measurements are compared to the predictions of Epos 1.99 [14], Sibyll 2.1 [21], Sibyll 2.3 [22], QGSJet II-04 [23] and Epos LHC [24]. The doubledifferential spectra as a function of $p$ and $p_{\mathrm{T}}$ can be found in Ref. [25] for the $\pi^{ \pm}$spectra and in Ref. [20] for the $\mathrm{K}^{ \pm}$ and $\mathrm{p}(\overline{\mathrm{p}})$ spectra.

\section{Production of $\Lambda, \bar{\Lambda}$ and $\mathrm{K}_{\mathrm{S}}^{0}$}

Since $\Lambda(\bar{\Lambda})$ and $\mathrm{K}_{\mathrm{S}}^{0}$ are neutral weakly decaying particles, they can be measured by NA61/SHINE through the detection of the charged particles which are produced in their decays. The invariant mass $\left(m_{\mathrm{inv}}\right)$ spectra for a given decay channel can then be used to extract their signal. The decay channels used here are $\Lambda \rightarrow p+\pi^{-}, \bar{\Lambda} \rightarrow \bar{p}+\pi^{+}$, 

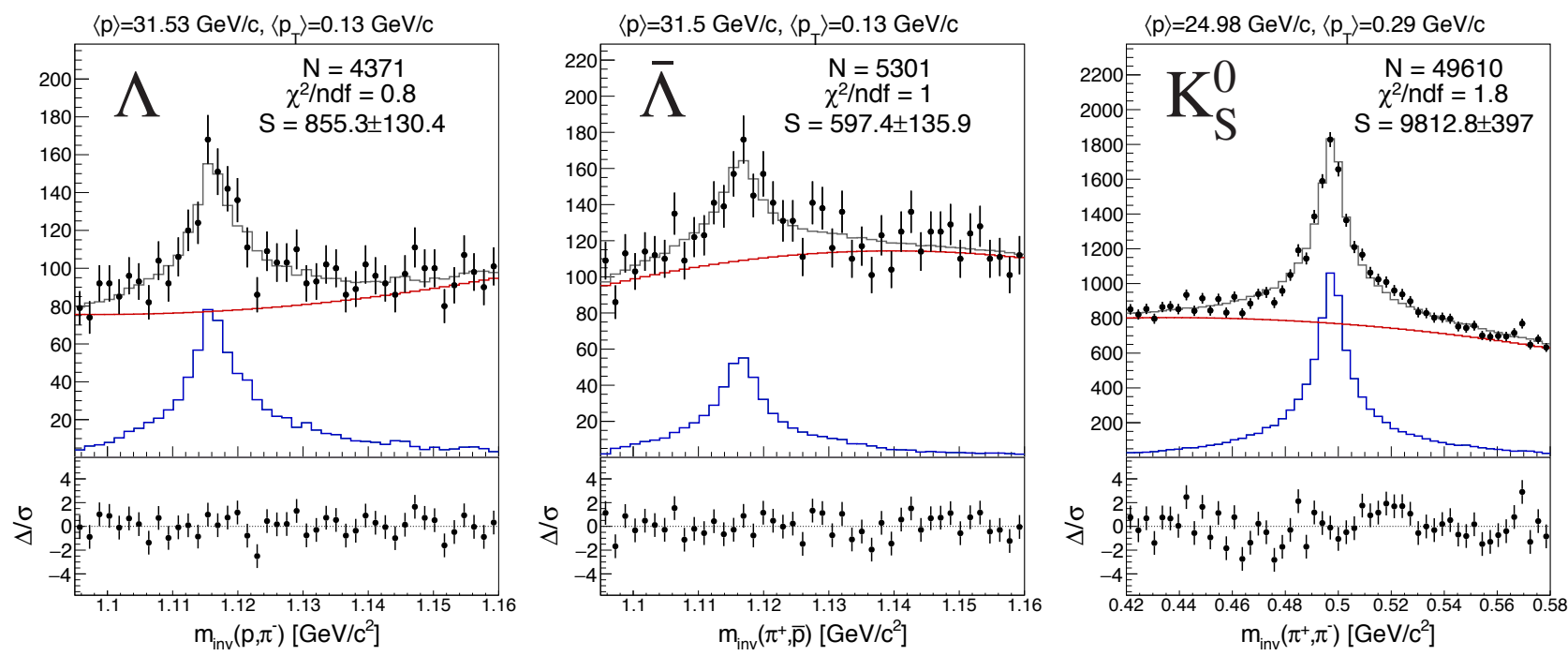

Figure 3: Example of the $m_{\text {inv }}$ distributions for one phase space bin $\left(\langle p\rangle\right.$ and $\left\langle p_{\mathrm{T}}\right\rangle \quad$ are indicated on the top of each plot $)$ of the $158 \mathrm{GeV} / c$ data set. The black markers show the measured distributions and the colored lines show the result of the signal extraction fit, where the signal is shown in blue and the background in red.

$K_{\mathrm{S}}^{0} \rightarrow \pi^{+}+\pi^{-}$. To extract the signal, the $m_{\text {inv }}$ distributions were fitted by considering a signal contribution, modeled by using Monte Carlo templates, and the background, modeled by a 2nd-degree polynomial function. Examples of the fitted $m_{\text {inv }}$ distributions are shown in Fig. 3.

This analysis was performed in 2-dimensional phase space bins of $p$ and $p_{\mathrm{T}}$. For each phase space bin, the detector effects were corrected by using Monte Carlo simulations. The full double-differential spectra as a function of $p$ and $p_{\mathrm{T}}$ for $\Lambda(\bar{\Lambda})$ and $\mathrm{K}_{\mathrm{S}}^{0}$ at 158 and $350 \mathrm{GeV} / c$ can be found in Ref. [26]. In Figs. 7 and 8 we show the measured single-differential spectra as function of $p$ (integrated over $\left.p_{\mathrm{T}}\right)$ together with predictions of the hadronic models.

\section{Production of $\rho^{0}, \mathbf{K}^{* 0}$ and $\omega$}

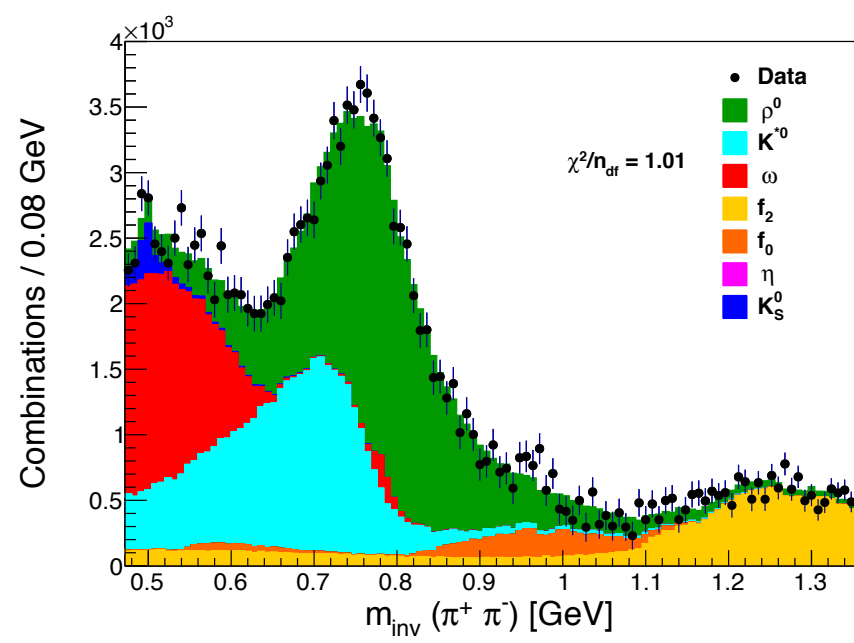

Figure 4: Example of the $m_{\text {inv }}\left(\pi^{+} \pi^{-}\right)$distribution for one $x_{\mathrm{F}}$ bin $\left(0.3<x_{\mathrm{F}}<0.4\right)$ of the $158 \mathrm{GeV} / c$ data set. The black markers show the measured distributions and the colored distributions show the results of the template fit.
By using the NA61/SHINE apparatus, the yields of $\rho^{0}$, $\mathrm{K}^{* 0}$ and $\omega$ can be measured through the $\pi^{+} \pi^{-}$invariant mass $\left(m_{\text {inv }}\left(\pi^{+} \pi^{-}\right)\right)$spectra. The signal extraction is performed by fitting Monte Carlo templates to the measured $m_{\text {inv }}\left(\pi^{+} \pi^{-}\right)$distribution. The Monte Carlo events were generated using Epos 1.99 as hadronic interaction model and they were passed through the full NA61/SHINE detector simulation and reconstruction chain. The estimation of the combinatorial background were done by two methods: the charge mixing method, in which the $\pi^{+} \pi^{+}$and $\pi^{-} \pi^{-}$are treated as the background, and the Monte Carlo method, in which the background mass distribution is obtained directly from simulations. One example of the $m_{\mathrm{inv}}\left(\pi^{+} \pi^{-}\right)$ distributions with the results of the template fit is shown in Fig. 4. After the signal extraction, the particle yields were corrected by the detector effects and the production spectra were derived. The full description of the analysis procedure and the results can be found in Ref. [27].

We show in Fig. 9 the obtained $\rho^{0}, \mathrm{~K}^{* 0}$ and $\omega$ spectra together with predictions from simulations with the hadronic models. The $\rho^{0}$ spectra are shown for both beam energies, 158 and $350 \mathrm{GeV} / c$, and the $\omega$ and $\mathrm{K}^{* 0}$ spectra are limited to the $158 \mathrm{GeV} / c$ data set because of the large uncertainties obtained at $350 \mathrm{GeV} / c$.

\section{Summary and conclusions}

The NA61/SHINE experiment, within its very rich program, has provided a large number of measurements which have been used for testing and tuning of hadronic interaction models used by the cosmic ray community. In this paper, we have summarized the results of the special cosmic ray runs for $\pi^{-}+\mathrm{C}$ interactions.

First, we have shown the identified spectra of charged hadrons obtained by using the $\mathrm{d} E / \mathrm{d} x$ measurements. Of particular interest here is the production spectra of $\mathrm{p}(\overline{\mathrm{p}})$, which are relevant to study the (anti)baryon productions in hadron-air interactions and its implications on the muon 
production in EAS. From the $\overline{\mathrm{p}}$ spectra shown in Figs. 5 and 6 , one can see that the (anti)baryon production is not underestimated in general by the models. In particular, the Epos model shows to describe very well the $\overline{\mathrm{p}}$ production. As a conclusion, the underproduction of (anti)baryons in $\pi$ air interactions by the hadronic models is unlikely to be the most relevant source of the lack of muons in simulations.

Secondly, we have shown the results of the $V^{0}$ analysis, aiming at the $\Lambda(\bar{\Lambda})$ and $\mathrm{K}_{\mathrm{S}}^{0}$ spectra. Although these measurements are surely relevant for model testing and tuning, our main motivation here is to reduce the systematic uncertainties on the $\pi^{ \pm}$and $\mathrm{p}(\overline{\mathrm{p}})$ spectra due to the feed-down contributions from weak decays. Since a significant fraction of $\pi^{ \pm}$and $\mathrm{p}(\overline{\mathrm{p}})$ detected are produced by the decay of $\Lambda, \bar{\Lambda}$ and $\mathrm{K}_{\mathrm{S}}^{0}$, this effect has to be corrected. In the results shown in Sec. 3 (and in Ref.[20]) this correction is done by using Monte Carlo simulations and the model dependence of this procedure is added to the systematic uncertainties. By measuring the spectra of $\Lambda, \bar{\Lambda}$ and $\mathrm{K}_{\mathrm{S}}^{0}$, we are able to avoid this model dependence and consequently reduce the systematic uncertainties. Updated $\pi^{ \pm}$and $\mathrm{p}(\overline{\mathrm{p}})$ spectra with improved systematic uncertainties will be presented in the future in another publication.

Finally, we have shown the final results of the meson resonance analysis which have already been published in Ref. [27]. From the $\rho^{0}$ production spectra shown in Fig. 9, one can see that none of the hadronic models can describe well the measurements. The small excess of $\rho^{0}$ observed with relation to the predictions from simulations can be relevant to explain the muon deficit in simulations.

\section{Acknowledgments}

We would like to thank the CERN EP, BE and EN Departments for the strong support of NA61/SHINE.

This work was supported by the Hungarian Scientific Research Fund (Grants NKFIH 123842-123959), the János Bolyai Research Scholarship of the Hungarian Academy of Sciences, the Polish Ministry of Science and Higher Education (grants 667/N-CERN/2010/0, NN 202484339 and NN 20223 1837), the Polish National Center for Science (grants 2011/03/N/ST2/03691, 2013/ 11/N/ST2/03879, 2014/13/N/ST2/02565, 2014/14/E/ST2/ 00018, 2014/15/B/ST2/02537 and 2015/18/M/ST2/00125, 2015/19/N/ST2 /01689, 2016/23/B/ST2/00692), the Russian Science Foundation, grant 16-12-10176, the Russian Academy of Science and the Russian Foundation for Basic Research (grants 08-02-00018, 09-02-00664 and 1202-91503-CERN), the Ministry of Science and Education of the Russian Federation, grant No. 3.3380.2017/4.6, the National Research Nuclear University MEPhI in the framework of the Russian Academic Excellence Project (contract No. 02.a03.21.0005, 27.08.2013), the Ministry of Education, Culture, Sports, Science and Technology, Japan, Grant-in-Aid for Scientific Research (grants 18071005, 19034011, 19740162, 20740160 and 20039012), the German Research Foundation (grant GA 1480/2-2), the Bulgarian Nuclear Regulatory Agency and the Joint Institute for Nuclear Research, Dubna (bilateral contract No. 44181-15/17), Bulgarian National Science Fund (grant DN08/ 11), Ministry of Education and Science of the Republic of
Serbia (grant OI171002), Swiss Nationalfonds Foundation (grant 200020117913/1), ETH Research Grant TH-01 07-3 and the U.S. Department of Energy.

\section{References}

[1] K.H. Kampert, M. Unger, Astroparticle Physics 35, 660 (2012), 1201.0018

[2] R. Engel, D. Heck, T. Pierog, Annual Review of Nuclear and Particle Science 61, 467 (2011)

[3] T. Abu-Zayyad et al. (HiRes/MIA Collaboration), Physical Review Letters 84, 4276 (2000), astro-ph/9911144

[4] A. Aab et al. (Pierre Auger Collaboration), Physical Review D91, 032003 (2015), 1408. 1421

[5] A. Aab et al. (Pierre Auger Collaboration), Physical Review Letters 117, 192001 (2016), 1610.08509

[6] A. Aab et al. (Pierre Auger Collaboration), Physical Review D90, 012012 (2014), 1407. 5919

[7] A. Aab et al. (Pierre Auger Collaboration), Physical Review D96, 122003 (2017), 1710.07249

[8] R.U. Abbasi et al. (Telescope Array Collaboration), Phys. Rev. D98, 022002 (2018), 1804 . 03877

[9] W.D. Apel et al. (KASCADE-Grande Collaboration), Astroparticle Physics 95, 25 (2017)

[10] H. Dembinski (IceCube Collaboration), EPJ Web of Conferences 145, 01003 (2017)

[11] J.A. Bellido, R.W. Clay, N.N. Kalmykov, I.S. Karpikov, G.I. Rubtsov, S.V. Troitsky, J. Ulrichs, Phys. Rev. D98, 023014 (2018), 1803.08662

[12] C. Meurer, J. Bluemer, R. Engel, A. Haungs, M. Roth, Czechoslovak Journal of Physics 56, A211 (2006), astro-ph/0512536

[13] I. C. Maris (for the NA61/SHINE Collaboration), Proc. of 31st ICRC (2009)

[14] T. Pierog, K. Werner, Physical Review Letters 101, 171101 (2008), astro-ph/0611311

[15] H.J. Drescher, Physical Review D77, 056003 (2008), Q712. 1517

[16] N. Abgrall et al. (NA61/SHINE Collaboration), Journal of Instrumentation 9, P06005 (2014), arXiv: 1401.4699

[17] N. Abgrall et al. (NA61/SHINE Collaboration), European Physics Journal C76, 84 (2016), 1510. 02703

[18] A. Aduszkiewicz et al. (NA61/SHINE Collaboration), European Physics Journal C77, 671 (2017), 1705.02467

[19] K. Abe et al. (T2K Collaboration), Nuclear Instruments and Methods in Physics. A659, 106 (2011), 1106.1238

[20] R. R. Prado (for the NA61/SHINE Collaboration), Proc. of 35th ICRC (2017), 1707.07902

[21] E.J. Ahn, R. Engel, T.K. Gaisser, P. Lipari, T. Stanev, Physical Review D80, 094003 (2009), 0906. 4113

[22] R. Engel, F. Riehn, A. Fedynitch, T.K. Gaisser, T. Stanev, Proc. of 35th ICRC (2017)

[23] S. Ostapchenko, Physical Review D83, 014018 (2011), 1010.1869 



Figure 5: Spectra of $\pi^{ \pm}, \mathrm{K}^{ \pm}$and $\mathrm{p}\left({ }^{-} \mathrm{p}\right)$ as a function of $p$ (integrated over $p_{\mathrm{T}}$ ), for the $158 \mathrm{GeV} / c$ data set. The statistical uncertainties are shown as black bars and the systematic ones as gray bands.

[24] T. Pierog, I. Karpenko, J.M. Katzy, E. Yatsenko, K. Werner, Physical Review C92, 034906 (2015), 1306.0121

[25] A. Herve (for the NA61/SHINE Collaboration), Proc. of 34th ICRC (2015), 1509. 06586
[26] R. R. Prado (for the NA61/SHINE Collaboration), in 7th International Conference on High Energy Physics in the LHC Era (Valparaiso, Chile, 2018)

[27] A. Aduszkiewicz et al. (NA61/SHINE Collaboration), European Physics Journal C77, 626 (2017), 1705.08206 



Figure 6: Spectra of $\pi^{ \pm}, \mathrm{K}^{ \pm}$and $\mathrm{p}\left({ }^{-} \mathrm{p}\right.$ ) as a function of $p$ (integrated over $p_{\mathrm{T}}$ ), for the $350 \mathrm{GeV} / c$ data set. The statistical uncertainties are shown as black bars and the systematic ones as gray bands.

\section{NA61/SHINE PRELIMINARY}
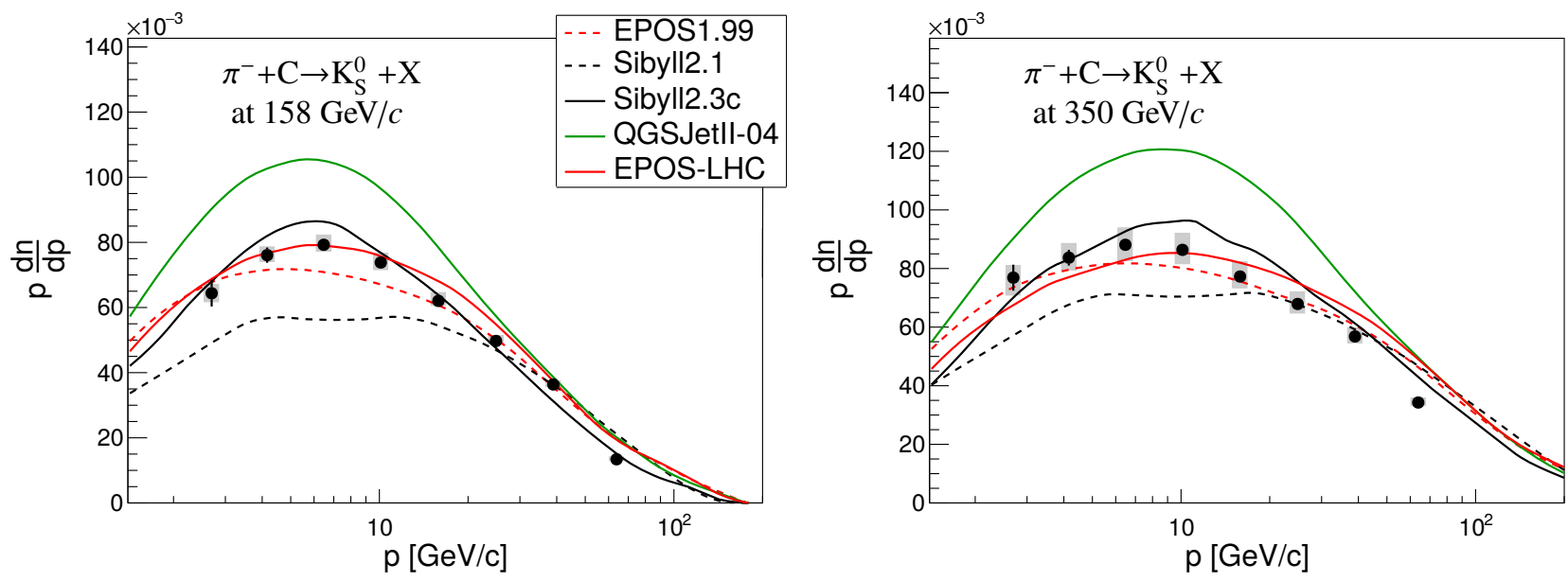

Figure 7: Spectra of $\mathrm{K}_{\mathrm{S}}^{0}$ as a function of $p$ (integrated over $p_{\mathrm{T}}$ ), for the 158 and $350 \mathrm{GeV} / c$ data set. The statistical uncertainties are shown as black bars and the systematic ones as gray bands. 

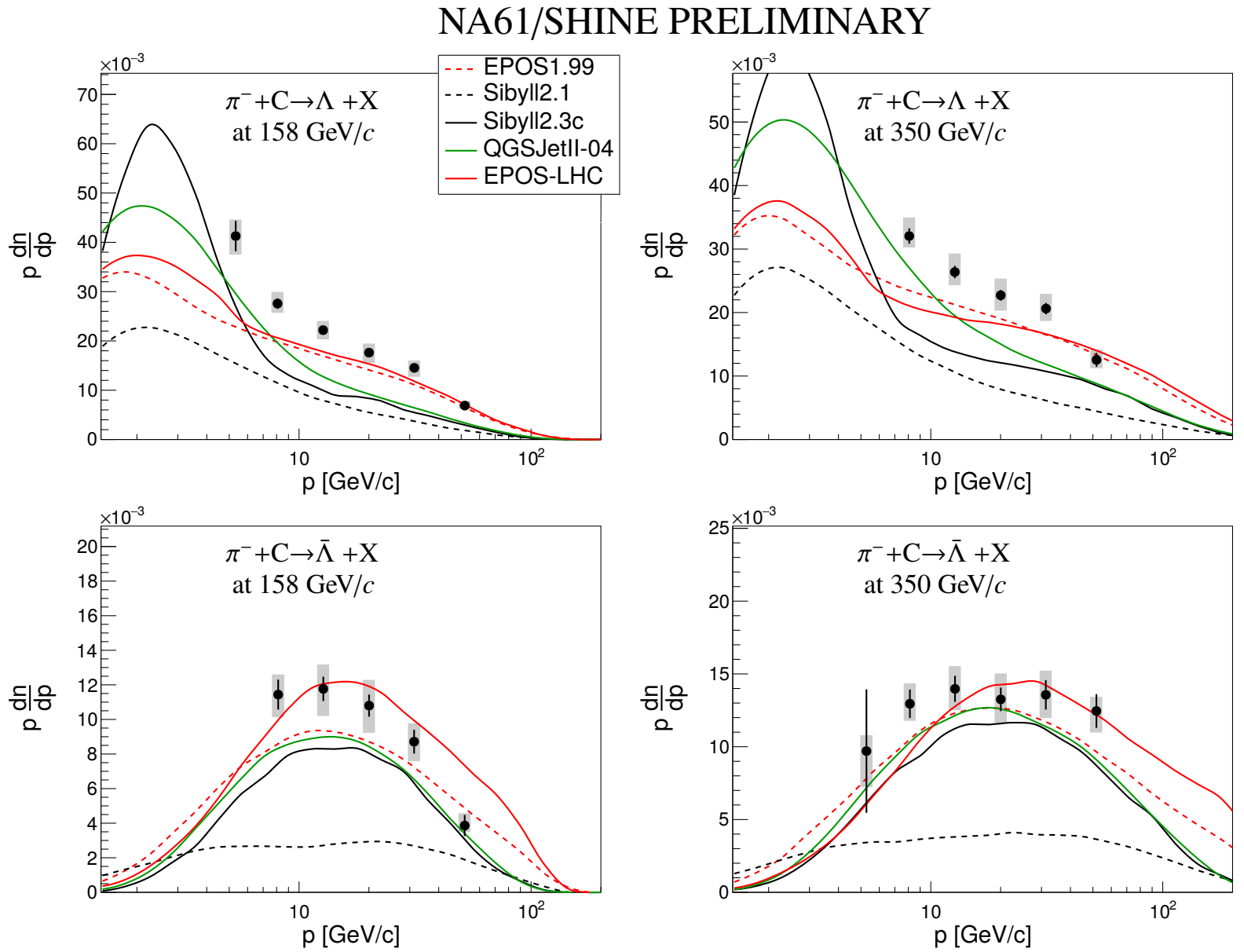

Figure 8: Spectra of $\Lambda\left(\Lambda^{-}\right.$) as a function of $p$ (integrated over $p_{\mathrm{T}}$ ), for the 158 and $350 \mathrm{GeV} / c$ data set. The statistical uncertainties are shown as black bars and the systematic ones as gray bands. 

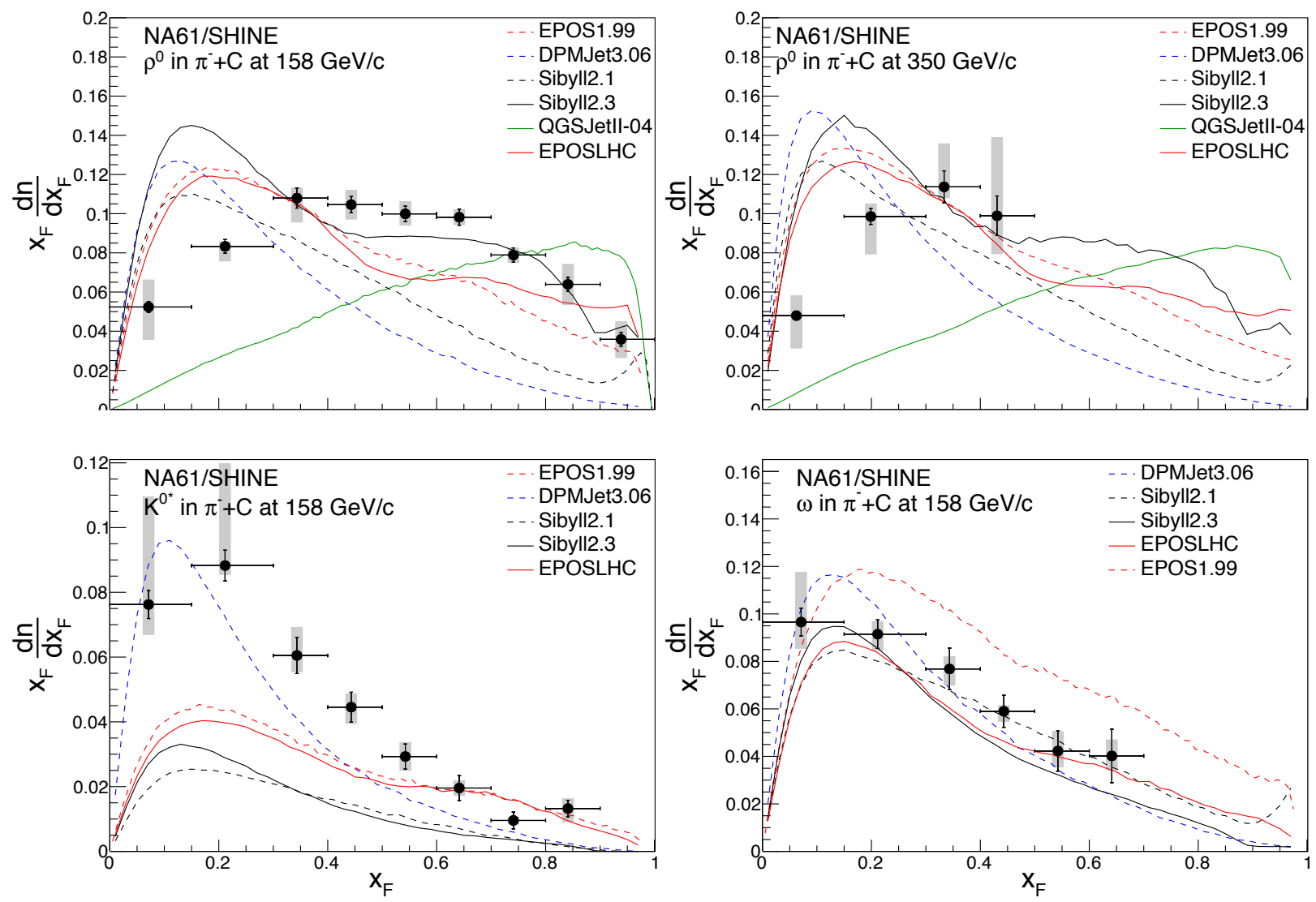

Figure 9: Spectra of $\rho^{0}, \mathrm{~K}^{* 0}$ and $\omega$ as a function of $x_{\mathrm{F}}$. The statistical uncertainties are shown as black bars and the systematic ones as gray bands. 